\title{
Effects of lapatinib on cell proliferation and apoptosis in NB4 cells
}

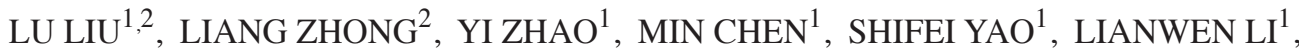 \\ CHUNLAN XIAO $^{1}$, ZHILING SHAN ${ }^{2}$, LIUGEN GAN ${ }^{1}$, TING XU ${ }^{1}$ and BEIZHONG LIU ${ }^{1,2}$ \\ ${ }^{1}$ Central Laboratory of Yong-Chuan Hospital, Chongqing Medical University, Chongqing 402160; \\ ${ }^{2}$ Key Laboratory of Laboratory Medical Diagnostics, Ministry of Education, \\ Department of Laboratory Medicine, Chongqing Medical University, Chongqing 400016, P.R. China
}

Received May 1, 2017; Accepted October 25, 2017

DOI: $10.3892 / \mathrm{ol} .2017 .7342$

\begin{abstract}
Acute promyelocytic leukemia (APL), characterized by the presence of the promyelocytic leukemia (PML)-retinoic acid $\alpha$ receptor $(\mathrm{RAR} \alpha)$ fusion protein, responds to treatment with all-trans retinoic acid (ATRA) and arsenic trioxide (ATO). However, drug resistance and side effects restrict the application of these reagents. Hence, the development of novel therapeutic drugs for APL treatment is critical. Lapatinib, a small-molecule tyrosine kinase inhibitor, has been used in the treatment of different tumors. However, it is unclear whether lapatinib exerts antitumor effects on APL. The present study investigated the antitumor effects and potential mechanisms of lapatinib on NB4 cells derived from APL. Cell Counting Kit-8 assay and colony forming analysis indicated that lapatinib inhibited NB4 cell proliferation in a dose-dependent manner. Flow cytometry analysis revealed that lapatinib induced cell cycle arrest at the $\mathrm{S}$ phase and promoted cell apoptosis. Furthermore, Liu's staining and Hoechst 33258 staining revelaed that lapatinib treatment induced an apoptotic nuclear phenomenon. Furthermore, lapatinib induced apoptosis by decreasing Bcl-2 and PML-RAR $\alpha$ levels, and by increasing the levels of Bax, cleaved PARP, cleaved caspase- 3 and cleaved caspase-9. In addition, lapatinib increased the levels of phospho-p38 MAPK and phospho-JNK, and decreased the levels of phospho-Akt. The p38 inhibitor PD169316 partially blocked lapatinib-induced proliferation inhibition and apoptosis, whereas the JNK inhibitor SP600125 had no such effects. Therefore, treatment with lapatinib may be a promising strategy for APL therapy.
\end{abstract}

\section{Introduction}

Acute promyelocytic leukemia (APL) is a subtype of acute myeloid leukemia (AML) that is characterized by

Correspondence to: Dr Beizhong Liu, Central Laboratory of Yong-Chuan Hospital, Chongqing Medical University, 1 Medical Road, Chongqing 402160, P.R. China

E-mail: liubeizhong@cqmu.edu.cn

Key words: lapatinib, proliferation, apoptosis, p38 MAPK, NB4 cells a disease-initiating chromosome translocation of $\mathrm{t}(15 ; 17)$ (q22:q21), leading to the generationof the promyelocytic leukemia (PML)-retinoic acid $\alpha$ receptor (RAR $\alpha$ ) fusion protein (1). At present, the treatment of APL involves the use of all-trans retinoic acid (ATRA) and arsenic trioxide (ATO). ATRA primarily induces differentiation, whereas ATO promotes apoptosis in APL cells (2). Despite the significant progress in therapeutic strategies, $10-30 \%$ patients with APL are still resistant to ATRA or ATO (3). Hence, the development of new therapeutic drugs for APL treatment is critical.

Lapatinib is a small-molecule, tyrosine kinase inhibitor that targets the epidermal growth factor receptor and human epidermal growth factor receptor (HER-2) (4,5). Clinically, lapatinib is mainly used for the treatment of HER-2 positive metastatic breast cancer, and is approved for use by the Food and Drug Administration of the USA $(1,6)$. In addition, since lapatinib is an oral drug and has few adverse reactions, it has been used in the treatment of several types of tumors, including triple-negative breast cancer, nasopharyngeal carcinoma, head and neck cancer, pancreatic cancer, cervical cancer, non-small cell lung cancer, and brain cancer (7-14). Moreover, several studies have reported the use of lapatinib in the treatment of leukemia; for example, lapatinib induces apoptosis in cell lines derived from acute myeloid leukemia (AML) and myelodysplastic syndrome (MDS) (15), and it has been shown to regulate autophagy, apoptosis , and megakaryocytic differentiation in K562 cells (16). In addition, lapatinib promotes autophagic cell death and differentiation in AML (17). Importantly, lapatinib inhibits cell proliferation and promotes cell apoptosis in Philadelphia chromosome-positive acute lymphoblastic leukemia (18). However, few studies have evaluated the effects of lapatinib in APL cells.

In the present study, we investigated the effects of lapatinib on NB4 cells, an APL cell line. We found that lapatinib inhibited cell proliferation and promoted apoptosis. Our observations suggested that lapatinib treatment may represent a novel therapeutic approach for the treatment of APL.

\section{Materials and methods}

Reagents. Lapatinib was purchased from Shanghai Selleck Chemicals Co., Ltd., (Shanghai, China). Liu's stain was purchased from BaSO Diagnostics Inc., (Zhuhai, China). 
The inhibitor of P-38MAPK PD169316 was purchased from MedChemExpress (New Jersey, USA). Hoechst 33258 and the JNK inhibitor SP600125 were purchased from Beyotime Institute of Biotechnology (Shanghai, China). Antibodies against caspase-3, caspase-9, cleaved caspase-9, p38MAPK, JNK, p-JNK, Akt, and p-Akt were purchased from Cell Signaling Technology, Inc., (Danvers, MA, USA). Antibodies against Bax, Bcl-2, and PARP were purchased from Wanleibio (Shenyang, China). Antibody against PML-RAR $\alpha$ was purchased from Abcam (Cambridge, MA, USA). Antibody against p-p38 MAPK was purchased from EMD Millipore (Billerica, MA, USA). Goat-anti-rabbit antibody, goat-anti-mouse antibody and anti- $\beta$-actin antibodies were purchased from Zhongshan Golden Brige Biotechnology Co., Ltd., (Beijing, China).

Cell culture. NB4 cells (Institutes for Biological Science, Shanghai, China) were cultured in RPIM 1640 (Gibco Life Technologies, Carlsbad, CA, USA) containing 10\% fetal bovine serum (Gibco Life Technologies) supplemented with penicllin $(100 \mathrm{mg} / \mathrm{ml})$ and streptomycin $(100 \mathrm{mg} / \mathrm{ml})$ at $37^{\circ} \mathrm{C}$ in an environment containing $5 \% \mathrm{CO}_{2}$. The medium was refreshed every day.

CCK-8 assay. NB4 cells were seeded in 96-well plates at a density of $1.0 \times 10^{4}$ cells/well. Then, cells were treated with different concentrations of lapatinib for $24 \mathrm{~h}$. After cultured 24 h, $10 \mu \mathrm{l}$ of CCK-8 (7Sea Cell Counting Kit; Sevenseas Futai Bio-technology Co., Ltd., Shanghai, China) was added to each well followed by incubation for $2 \mathrm{~h}$ at $37^{\circ} \mathrm{C}$. Cell viability was assessed by detection of absorbance at $450 \mathrm{~nm}$ using a spectrophotometer (Bio-Rad Laboratories, Inc., Hercules, CA, USA). The experiment was repeated at least three times.

Colony forming assay. NB4 cells were exposed to lapatinib or DMSO for $24 \mathrm{~h}$. Then, cells were plated in 24-well plates in methylcellulose medium in triplicate. The numbers of colonies were determined following incubation for 14 days at $37^{\circ} \mathrm{C}$ and $5 \% \mathrm{CO}_{2}$.

Flow cytometry analysis. NB4 cells were fixed with pre-cold $70 \%$ ethanol overnight at $4^{\circ} \mathrm{C}$ and washed with PBS. Then, incubated with $50 \mu \mathrm{l}$ Propidium Iodide (PI) for $15 \mathrm{~min}$ at room temperature and cells were analysis in flow cytometer. Each experiment was repeated at least three times.

NB4 cells were treated of various concentrations of lapatinib for $24 \mathrm{~h}$, and cells were harvested by centrifugation at $3000 \mathrm{r}$ for $5 \mathrm{~min}$. After washed three times with pre-cold PBS, cells were re-suspended in Binding Buffer (Sungene Biotech Co., Ltd., Tianjing, China), and stained by Annexin V-FITC and PI (Sigma-Aldrich, St. Louis, MO, USA) for $15 \mathrm{~min}$ at room temperature and cells were analysis in flow cytometer. Each experiment was repeated at least three times.

Liu's staining. NB4 cells were treated with different concentrations of lapatinib for $24 \mathrm{~h}$. Cells in each group were collected and plated onto the glass slides. First, Liu A was added to the sample spot for $20 \mathrm{~s}$ and mixed with twice the volume of Liu B for another $30 \mathrm{~s}$. Then, the slides were washed with water and air dried for observation using a microscope.
Hoechst 33258 staining. NB4 cells were treated with different concentrations of lapatinib for $24 \mathrm{~h}$. Cells in each group were collected and plated onto the glass slides, followed by fixative in $4 \%$ para-formaldehyde at room temperature. After $20 \mathrm{~min}$, cells were washed twice with PBS, stained with Hoechst 33258 for $10 \mathrm{~min}$ and then washed twice with PBS. Nuclear morphological changes in the cells were observed under fluorescence microscope.

Western blot analysis. NB4 cells were lysed in radioimmunoprecipitation solution containing protease inhibitor phenylme-thanesulfonyl fluoride (PMSF), phosphatase inhibitor $\mathrm{Na} \mathrm{F}$ and Na3VO3. Protein concentration was determined with BCA method. A total of $50 \mu \mathrm{g}$ of proteins were separated by $10 \%$ SDS-PAGE and transferred to polyvinylidence difluoride membrane. Membranes were blocked with 5\% non-fat milk for $2 \mathrm{~h}$, and incubated with specific antibodies $(1: 1,000)$ over night at $4^{\circ} \mathrm{C}$. Then, membranes were incubated with goat-anti-rabbit and goat-anti-mouse secondary antibodies $(1: 4,000)$ for $1 \mathrm{~h}$ at $37^{\circ} \mathrm{C}$. After washing with Tris-buffered saline containing Tween-20 (TBST), the immunoreactive complexes were visualized using an enhanced chemiluminescence system. Protein bands were analyzed by Quantity One Software (Bio-Rad Laboratories, Inc.). Each experiment was repeated at least three times.

Statistical analysis. Data were expressed as means \pm standard deviation (SD). Statistical analysis was performed with SPSS version 17.0. An independent samples t-test was used to compare the results of two groups. Statistical analysis of western blot results was performed using analysis of variance (ANOVA). $\mathrm{P}<0.05$ was considered to indicate a statistically significant difference. Each experiment was repeated at least three times.

\section{Results}

Lapatinib inhibits the proliferation of NB4 cells. The effect of lapatinib on the viability of NB4 cells was evaluated using CCK-8 assays. Treatment of NB4 cells with 0-20 $\mu \mathrm{M}$ lapatinib for $24 \mathrm{~h}$ led to a concentration-dependent reduction in cell viability (Fig. 1A). Based on these observations, 15 and $20 \mu \mathrm{M}$ lapatinib were selected for our study. Exposure to 15 and $20 \mu \mathrm{M}$ lapatinib visibly inhibited the colony-forming capacity of NB4 cells compared to the cells of the dimethyl sulfoxide (DMSO) group (Fig. 1B).

Lapatinib induces cell cycle arrest at the $S$ phase. The cell cycle of NB4 cells was detected by a flow cytometric assay. Compared to the cell cycle profile of the DMSO treated group, 15 and $20 \mu \mathrm{M}$ lapatinib treatment dramatically increased the percentage of cells in the $\mathrm{S}$ phase (Fig. 2).

Lapatinib promotes apoptosis in NB4 cells. NB4 cells were treated with 15 and $20 \mu \mathrm{M}$ lapatinib for $24 \mathrm{~h}$ and cell apoptosis was detected using flow cytometry. As shown in Fig. 3, cell apoptosis increased in the lapatinib treated groups compared to that of the DMSO-treated group (Fig. 3).

Effects of lapatinib on NB4 cell morphology. To further assess the effect of lapatinib treatment on NB4 cells' morphology, 

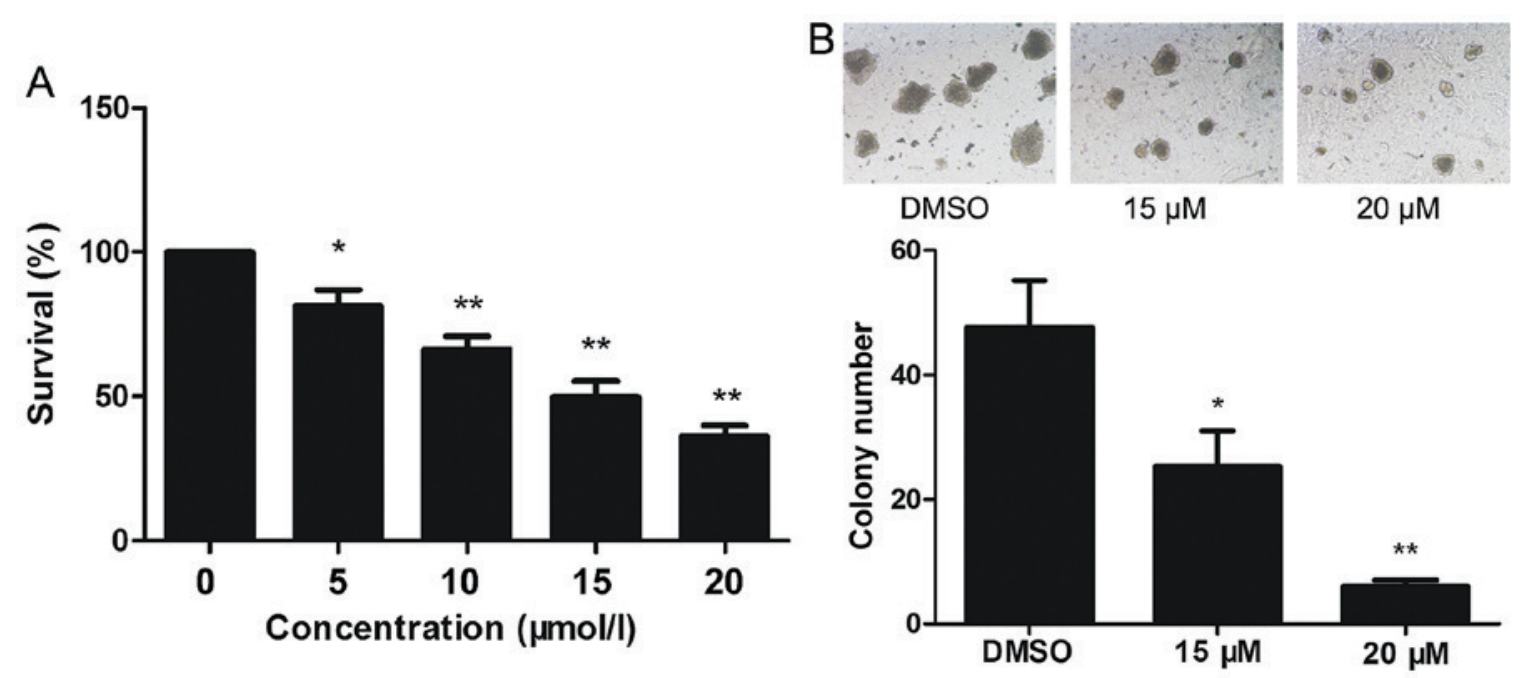

Figure 1. Lapatinib inhibited the proliferation of NB4 cells. (A) NB4 cells were cultured with 0-20 $\mu \mathrm{M}$ lapatinib for $24 \mathrm{~h}$ in 96 -well plate. Cytotoxicity was detected by CCK-8 assays. (B) The colony-forming ability of NB4 cells was tested with or without the treatment of lapatinib, the magnification is 40x. Data was expressed as means $\pm \mathrm{SD} .{ }^{*} \mathrm{P}<0.05,{ }^{* *} \mathrm{P}<0.01$.
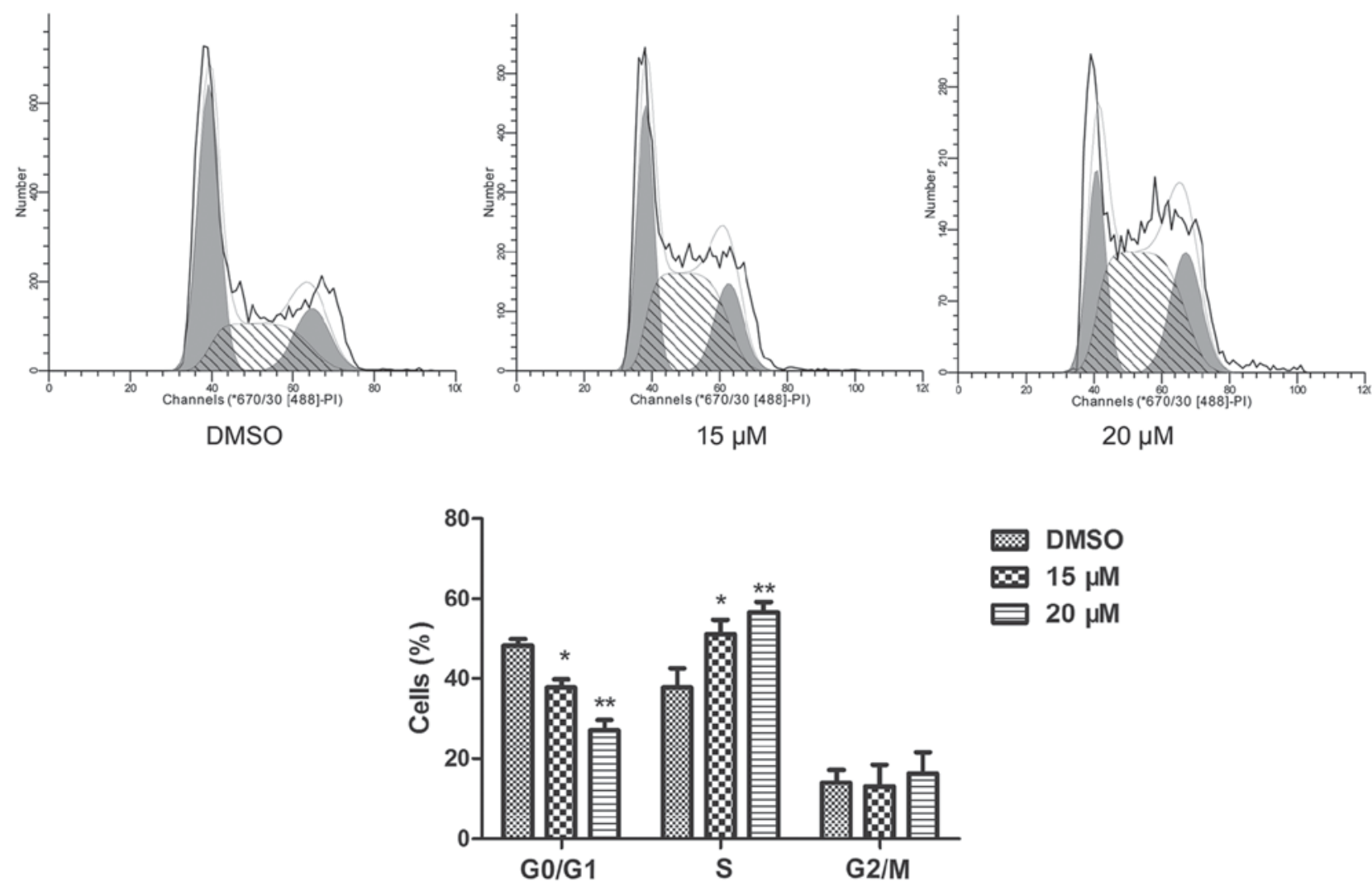

Figure 2. Lapatinib induced S phase arrest in NB4 cells. NB4 cells were treated with 15 and $20 \mu \mathrm{M}$ lapatinib for 24 h, cells were harvested for cell cycle analysis by flow cytometry. Data was expressed as means $\pm \mathrm{SD} .{ }^{*} \mathrm{P}<0.05,{ }^{* *} \mathrm{P}<0.01$.

cells were stained with Liu's stain after treatment with 15 and $20 \mu \mathrm{M}$ lapatinib. Results showed that nuclear fragmentation was observed in the lapatinib-treated group (Fig. 4A). In addition, after incubation with 15 and $20 \mu \mathrm{M}$ lapatinib, cells were harvested and stained with Hoechst 33258. The nuclear fluorescence intensities of the lapatinib treated groups were stronger than that of the negative control group (Fig. 4B), and nuclear fragmentation and condensation were observed in the lapatinib- treated group (Fig. 4).
Effects of lapatinib on apoptosis-related protein in NB4 cells. To investigate the effect of lapatinib on apoptosis in NB4 cells, the levels of apoptosis-related proteins were detected by western blotting. Bcl-2 was notably down-regulated with lapatinib treatment. In addition, the expression of Bax, cleaved caspase-3, cleaved caspase-9, and cleaved PARP were up-regulated in the lapatinib group but not in the DMSO group (Fig. 5A). The level of the fusion protein PML-RAR $\alpha$ was down-regulated with lapatinib treatment (Fig. 5B). 

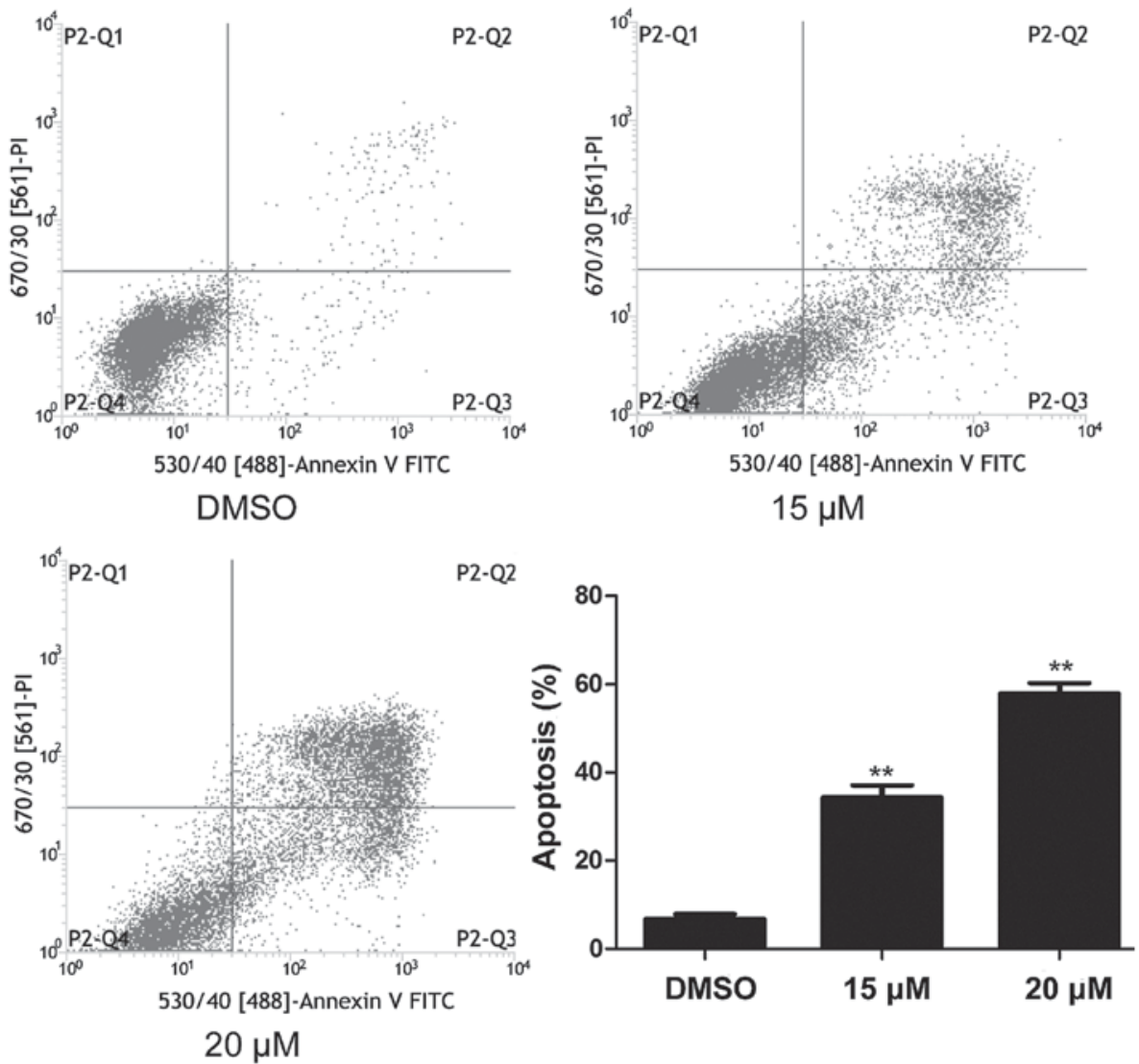

Figure 3. Lapatinib induced apoptosis of NB4 cells. Cells were treated with 15 and $20 \mu \mathrm{M}$ lapatinib for $24 \mathrm{~h}$, and apoptosis was analyzed by flow cytometry using double staining with FITC-labeled annexin-V and PI. Cells undergoing early apoptosis are Annexin V-FITC $/ \mathrm{PI}^{-}$, while cells were undergoing late apoptosis are Annexin V-FITC $/ \mathrm{PI}^{+}$. The percentages of late and late apoptotic cells were summed to give the total number of apoptotic cells. Data was expressed as means $\pm \mathrm{SD} .{ }^{* * *} \mathrm{P}<0.01$.
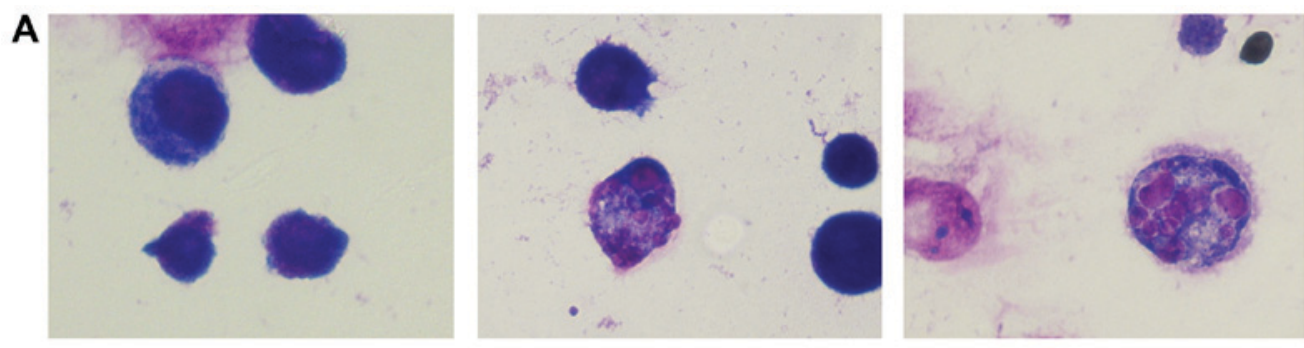

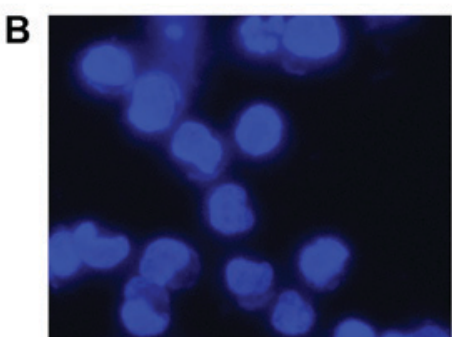

DMSO

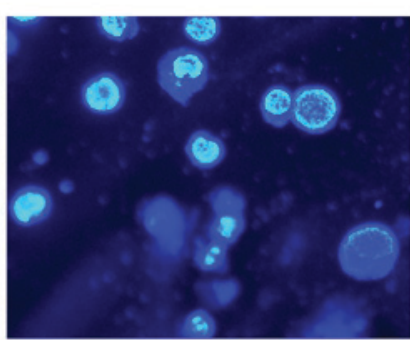

$15 \mu \mathrm{M}$

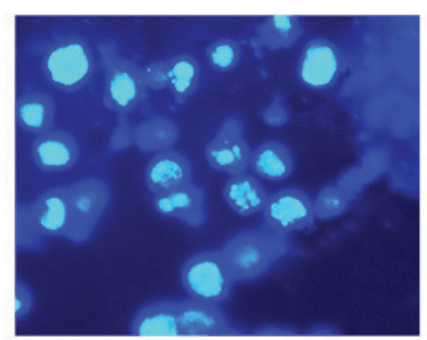

$20 \mu \mathrm{M}$

Figure 4. Effects of lapatinib on NB4 cell morphology. Cells were treated with 15 and $20 \mu \mathrm{M}$ lapatinib for $24 \mathrm{~h}$. (A) Cells stained with Liu's staining were imaged with a microscope, the magnification is 1,000x. (B) Cells stained with Hoechst 33258 were imaged with a fluorescence microscope, the magnification is $400 x$.

Effects of lapatinib on MAPKs and AKT signaling pathways. To investigate the molecular mechanisms of lapatinib action on NB4 cells, we analyzed the levels of Akt, p-Akt, p38MAPK, p-p38MAPK, JNK, and p-JNK. Results showed that lapatinib notably down-regulated the expression of
p-Akt and up-regulated the expression of p-p38MAPK and p-JNK (Fig. 6).

PD169316 weakens the effect of lapatinib in NB4 cells. To further study the involvement of p38MAPK and JNK signaling 
A
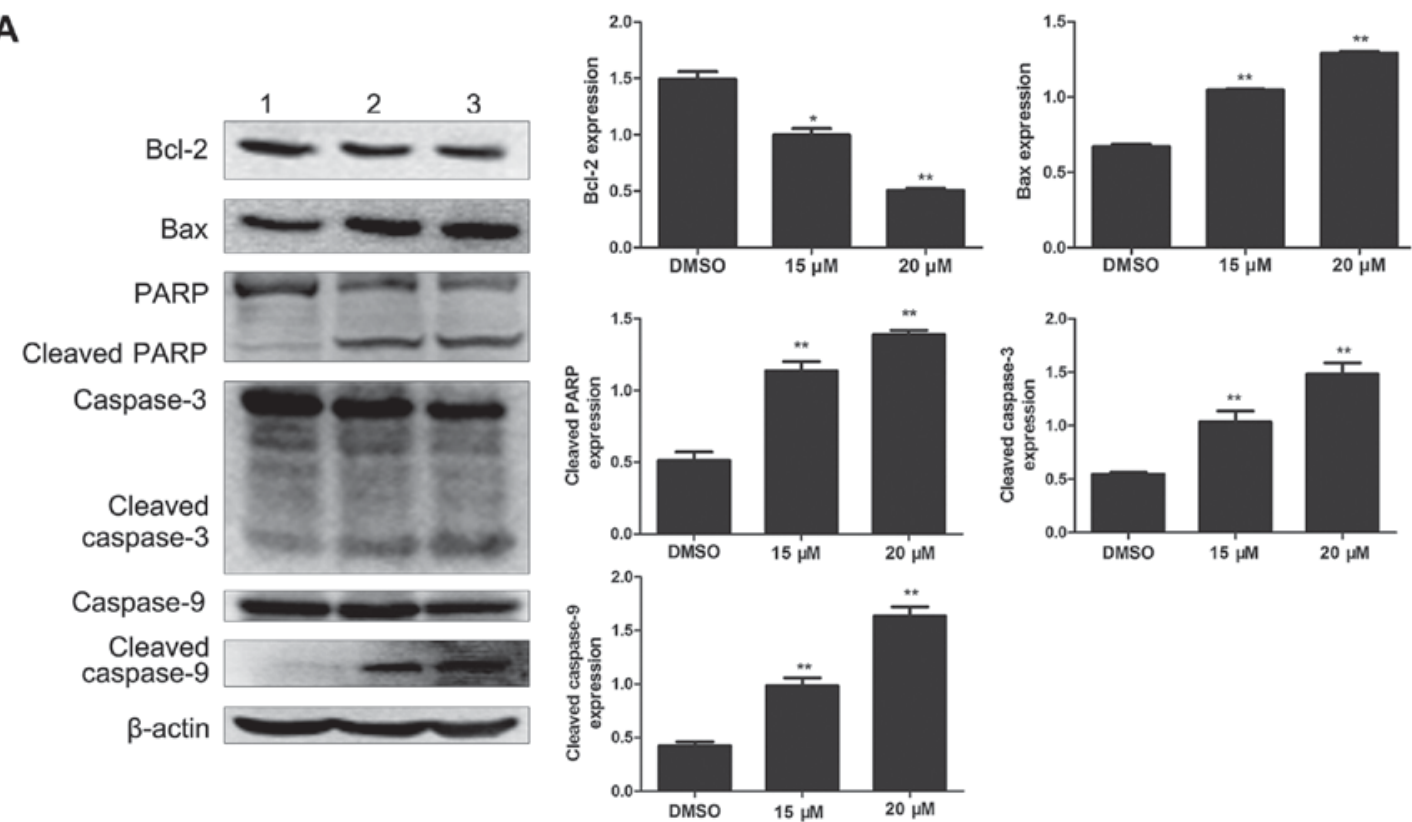

B
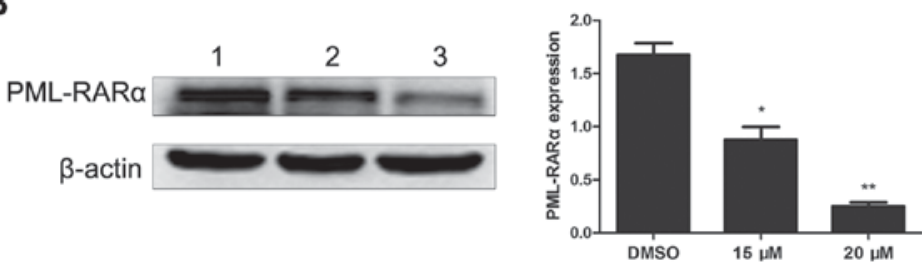

Figure 5. Protein expression levels in NB4 cells. Cells were treated with 15 and $20 \mu \mathrm{M}$ lapatinib for $24 \mathrm{~h}$. (A) The levels of Bax, Bcl-2, PARP, caspase-3/9, and cleaved caspase-3/9 proteins were measured by western blotting. (B) The expression of PML-RAR $\alpha$. Quantification analysis of western blotting, $\beta$-actin was served as a control. Data was expressed as means \pm SD. $P<0.05,{ }^{* *} \mathrm{P}<0.01$ (1 NB4 cells treated with DMSO; 2 NB4 cells treated with $15 \mu$ M lapatinib; 3 NB4 cells treated with $20 \mu \mathrm{M}$ lapatinib).
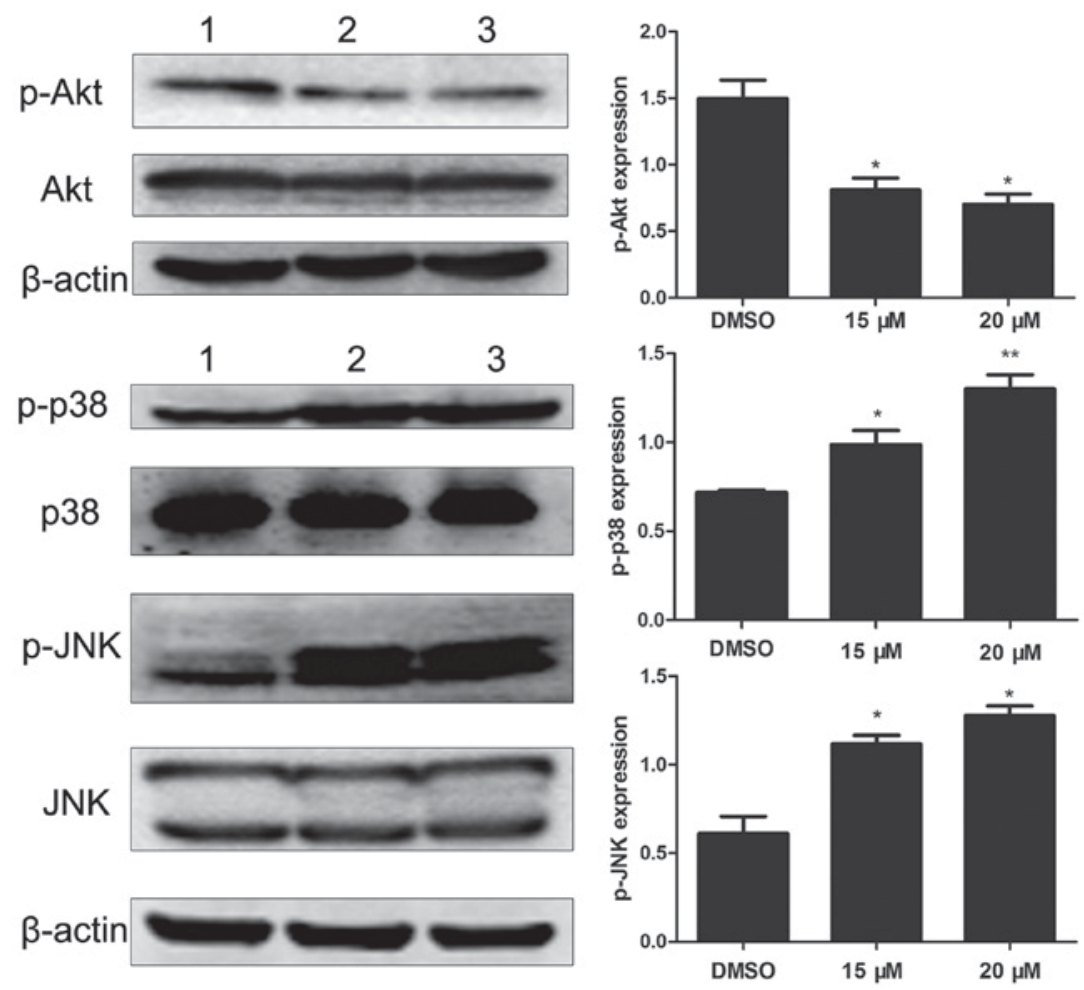

Figure 6. Effects of lapatinib on MAPKs and PI3K/AKT signaling pathways. Cells were treated with 15 and $20 \mu \mathrm{M}$ lapatinib for $24 \mathrm{~h}$, the expression of p-AKT, AKT, p-p38 MAPK, p38 MAPK, p-JNK, and JNK were measured by western blotting. Quantification analysis of western blotting, $\beta$-actin was served as a control. Data were expressed as means \pm SD. ${ }^{*} \mathrm{P}<0.05,{ }^{* *} \mathrm{P}<0.01$ (A: 1 DMSO; $215 \mu \mathrm{M}$ lapatinib; $320 \mu \mathrm{M}$ lapatinib. D: 1 DMSO; 2 PD169316; $315 \mu \mathrm{M}$ lapatinib; 4 PD169316+15 $\mu \mathrm{M}$ lapatinib). 

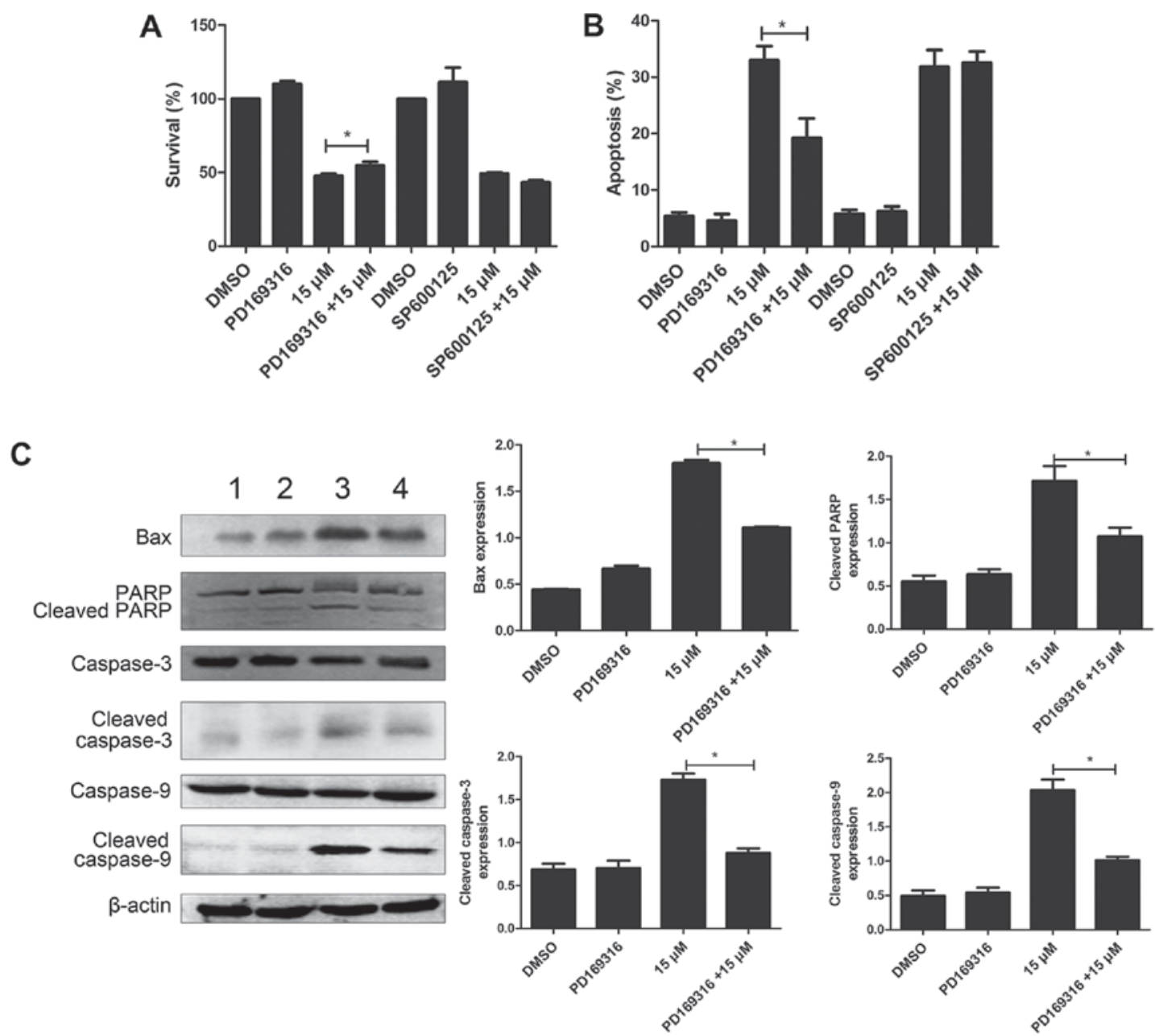

Figure 7. PD169316 reduces cell proliferation inhibition and apoptosis induced by lapatinib. NB4 cells were treated with $15 \mu \mathrm{M}$ lapatinib for $24 \mathrm{~h}$ in the presence or absence of PD169316 or SP600125. (A) Cell viability was measured by CCK-8 assay. (B) Cell apoptosis was analyzed by flow cytometry. (C) The levels of Bax, PARP, cleaved caspase-3/9, and caspase-3/9 were measured by western blotting. Quantification analysis of western blotting, $\beta$-actin was served as a control. Data was expressed as means $\pm \mathrm{SD}$. "P<0.05 (A: 1 DMSO; $215 \mu \mathrm{M}$ lapatinib; $320 \mu \mathrm{M}$ lapatinib. D: 1 DMSO; 2 PD169316; $315 \mu \mathrm{M}$ lapatinib; 4 PD169316+15 $\mu \mathrm{M}$ lapatinib).

pathways, in the mode of action of lapatinib, we treated the cells with $10 \mu \mathrm{M}$ each of PD169316 or SP600125 for 30 min before incubating with $15 \mu \mathrm{M}$ lapatinib. The results of the CCK-8 assay showed that PD169316 could partially reverse the growth inhibition induced by lapatinib treatment. FCM detections demonstrated that PD169316 partially reduced lapatinib-induced apoptosis. However, the JNK inhibitor, SP600125, had no such effect (Fig. 7A and B). In addition, the levels of Bax, cleaved caspase-3, cleaved caspase-9, and cleaved PARP were significantly reduced upon treatment with a combination of lapatinib and PD169316 than with lapatinib treatment alone (Fig. 7C).

\section{Discussion}

Previous studies showed that lapatinib decreased the viability of MOLM-13 and HL-60 cells (15), head and neck squamous carcinoma cells (19), gastric cancer cells (20), and breast cancer cells (21). In this study, the CCK-8 assay showed that proliferation of NB4 cells was inhibited by 5-20 $\mu \mathrm{M}$ lapatinib in a concentration-dependent manner. Colony-forming assays showed that 15 and $20 \mu \mathrm{M}$ lapatinib dramatically reduced colony formation, indicating that lapatinib could suppress NB4 cell proliferation. Moreover, some recent studies have reported that lapatinib promotes cell cycle arrest in the $\mathrm{G}_{0} / \mathrm{G}_{1}$ phase (19-21). However, our results showed that lapatinib reduced the number of cells in the $G_{1}$ phase and increased the number of cells in the $\mathrm{S}$ phase. Based on these results, we hypothesize that lapatinib inhibits cell proliferation by inducing $S$ phase arrest.

Apoptosis is a process of programmed cell death and involves balancing of pro-survival and pro-cell death functions in the cell (22). Apoptosis is characterized by nuclear condensation, nuclear fragmentation, and formation of apoptotic bodies. Additionally, caspase-3 and PARP are cleaved during activation of apoptosis (23-25). In our study, flow cytometry analysis showed that lapatinib promoted cell apoptosis; indeed, nuclear fragmentation, nuclear condensation, and the presence of few apoptotic bodies were observed in NB4 cells after lapatinib treatment, and lapatinib induced cleavage of caspase-3 and PARP in a dose-dependent manner. Two apoptotic pathways exist: the extrinsic apoptotic pathway involving the death receptors is regulated by caspase-8, whereas the intrinsic apoptotic pathway involving the mitochondria is related to activation of caspase- 3 and caspase-9 (26). Our data showed that treatment with lapatinib 
induced the activation of caspase- 9 rather than caspase- 8 (data not shown); thus, we assumed that lapatinib may induce apoptosis via the intrinsic apoptotic pathway in NB4 cells. Indeed, our analysis showed that lapatinib decreased Bcl-2 and increased Bax levels, thereby demonstrating that lapatinib induces apoptosis through the intrinsic pathway mediated by the Bcl-2 family, which involves Bcl-2 and Bax. Interestingly, lapatinib also reduce the level of PML-RAR $\alpha$, which blocks granulocyte cell maturation. This result showed that lapatinib-induced apoptosis was associated with the degradation of PML-RAR $\alpha$. Taken together, our results suggested that lapatinib treatment may represent a therapeutic option for APL, although further studies are required to fully elucidate the mechanisms involved in this process.

MAPK family members can be activated by different factors and have important roles in apoptosis. MAPK signaling pathways, including ERK, p38 MAPK, and JNK, have been identified as chemotherapeutic targets for sensitizing cancer cells for apoptosis (27). Typically, p38 kinase and JNK are known as cell death signals, whereas ERK is a survival signal (28). Previous studies have shown that lapatinib induces apoptosis in breast cancer cells through the STAT5 and JNK signaling pathways (29). Indeed, after treatment with lapatinib, cells showed increased phosphorylation of p38 MAPK and JNK in a concentration-dependent manner. In addition, the p38 inhibitor PD169316 reduced the lapatinib-induced inhibition of cell viability and apoptosis, whereas the JNK inhibitor SP600125 did not affect these processes. Moreover, co-treatment with lapatinib and a p38 inhibitor significantly decreased the levels of Bax, cleaved PARP, cleaved caspase-3 and cleaved caspase- 9 compared to those in cells treated with lapatinib alone. These results suggested that the p38 MAPK pathway was involved in lapatinib-induced apoptosis. In addition to the MAPK pathway, the PI3K/AKT signal transduction pathway plays a vital role in cell survival and prevents cancer cells from undergoing apoptosis during tumorigenesis (30). Several studies have reported that lapatinib induces apoptosis in triple-negative breast cancer cells by inhibiting the CIP2A/PP2A/P-AKT signaling pathways (11). Additionally, lapatinib can potentiate radiation-induced cell death in HER-2-overexpressing breast cancer cells via reduction of p-AKT levels (31). Consistent with this observation, our results showed that lapatinib exerted significant inhibitory effects on p-AKT levels. These results suggested that the AKT pathway was involved in lapatinib-induced apoptosis in NB4 cells.

Previous studies have reported that lapatinib induces autophagic cell death in acute myeloblastic leukemia-derived cell lines (17); however, we did not detect any changes in the levels of autophagy markers ATG5, ATG7, and LC3II following lapatinib treatment. These contradictory results could be attributed to variations in the concentrations of lapatinib, differences in the time points used in the experiments, cell status, and variations in other experimental factors.

In conclusion, our present study indicated that lapatinib inhibited NB4 cell growth and caused cell cycle arrest, and promoted apoptosis potentially through the p38MAPK and AKT signaling pathways. These results suggested that treatment with lapatinib may be an effective strategy for APL therapy. Further investigations are required for developing novel therapeutic approaches for the treatment of leukemia.

\section{Acknowledgements}

The present study was supported by the National Natural Science Foundation of China (grant no. 81171658) and the Natural Science Foundation Project of CQ CSTC (grant no. 2011BA5037).

\section{References}

1. $\mathrm{Ma} \mathrm{H}$ and Yang J: Insights into the all-trans-retinoic acid and arsenic trioxide combination treatment for acute promyelocytic leukemia: A meta-analysis. Acta Haematol 134: 101-108, 2015.

2. Brown $G$ and Hughes P: Retinoid differentiation therapy for common types of acute myeloid leukemia. Leuk Res Treatment 2012: 939021, 2012.

3. Kawasaki K, Akaike H, Miyauchi A and Ouchi K: Sivelestat relieves respiratory distress refractory to dexamethasone in all-trans retinoic acid syndrome: A report of two cases. Eur J Haematol 77: 448-452, 2006.

4. Ito Y, Tokudome N, Sugihara T, Takahashi S and Hatake K: Does lapatinib, a small-molecule tyrosine kinase inhibitor, constitute a breakthrough in the treatment of breast cancer? Breast cancer 14: 156-162, 2007.

5. Kopper L: Lapatinib: A sword with two edges. Pathol Oncol Res 14: 1-8, 2008.

6. Konecny GE, Pegram MD, Venkatesan N, Finn R, Yang G, Rahmeh M, Untch M, Rusnak DW, Spehar G, Mullin RJ, et al: Activity of the dual kinase inhibitor lapatinib (GW572016) against HER-2-overexpressing and trastuzumab-treated breast cancer cells. Cancer Res 66: 1630-1639, 2006.

7. Beck TN, Georgopoulos R, Shagisultanova EI, Sarcu D, Handorf EA, Dubyk C, Lango MN, Ridge JA, Astsaturov I, Serebriiskii IG, et al: EGFR and RB1 as dual biomarkers in HPV-negative head and neck cancer. Mol Cancer Ther 15: 2486-2497, 2016

8. Gore J, Imasuen-Williams IE, Conteh AM, Craven KE, Cheng $M$ and Korc $M$ : Combined targeting of TGF- $\beta$, EGFR and HER2 suppresses lymphangiogenesis and metastasis in a pancreatic cancer model. Cancer Lett 379: 143-153, 2016.

9. Hsiao YC, Yeh MH, Chen YJ, Liu JF, Tang CH and Huang WC: Lapatinib increases motility of triple-negative breast cancer cells by decreasing miRNA-7 and inducing Raf-1/MAPK-dependent interleukin-6. Oncotarget 6: 37965-37978, 2015.

10. Jin HO, Hong SE, Kim CS, Park JA, Kim JH, Kim JY, Kim B, Chang YH, Hong SI, Hong YJ, et al: Combined effects of EGFR tyrosine kinase inhibitors and vATPase inhibitors in NSCLC cells. Toxicol Appl Pharmacol 287: 17-25, 2015.

11. Liu CY, Hu MH, Hsu CJ, Huang CT, Wang DS, Tsai WC, Chen YT, Lee CH, Chu PY, Hsu CC, et al: Correction: Lapatinib inhibits CIP2A/PP2A/p-Akt signaling and induces apoptosis in triple negative breast cancer cells. Oncotarget 8: 10760, 2017.

12. Liu L, Huang P, Wang Z, Chen N, Tang C, Lin Z and, Peng P: Inhibition of eEF-2 kinase sensitizes human nasopharyngeal carcinoma cells to lapatinib-induced apoptosis through the Src and Erk pathways. BMC Cancer 16: 813, 2016.

13. Oh DY, Kim S, Choi YL, Cho YJ, Oh E, Choi JJ, Jung K, Song JY, Ahn SE, Kim BG, et al: HER2 as a novel therapeutic target for cervical cancer. Oncotarget 6: 36219-36230, 2015.

14. Booth L, Cruickshanks N, Ridder T, Chen CS, Grant S and Dent P: OSU-03012 interacts with lapatinib to kill brain cancer cells. Cancer Biol Ther 13: 1501-1511, 2012.

15. Lainey E, Thépot $\mathrm{S}$, Bouteloup $\mathrm{C}$, Sébert M, Adès L, Tailler M, Gardin C, de Botton S, Baruchel A, Fenaux P, et al: Tyrosine kinase inhibitors for the treatment of acute myeloid leukemia: delineation of anti-leukemic mechanisms of action. Biochem Pharmacol 82: 1457-1466, 2011.

16. Huang HL, Chen YC, Huang YC, Yang KC, Pan Hy, Shih SP and Chen YJ: Lapatinib induces autophagy, apoptosis and megakaryocytic differentiation in chronic myelogenous leukemia K562 cells. PLoS One 6: e29014, 2011.

17. Chen YJ, Fang LW, Su WC, Hsu WY, Yang KC and Huang HL: Lapatinib induces autophagic cell death and differentiation in acute myeloblastic leukemia. Onco Targets Ther 9: 4453-4464, 2016. 
18. Irwin ME, Nelson LD, Santiago-O'Farrill JM, Knouse PD, Miller CP, Palla SL, Siwak DR, Mills GB, Estrov Z, Li S, et al: Small molecule ErbB inhibitors decrease proliferative signaling and promote apoptosis in philadelphia chromosome-positive acute lymphoblastic leukemia. PLoS One 8: e70608, 2013.

19. Fumagalli I, Dugue D, Bibault JE, Clémenson C, Vozenin MC, Mondini M and Deutsch E: Cytotoxic effect of lapatinib is restricted to human papillomavirus-positive head and neck squamous cell carcinoma cell lines. Onco Targets Ther 8: 335-345, 2015.

20. Oshima Y, Tanaka H, Murakami H, Ito Y, Furuya T, Kondo E, Kodera $Y$ and Nakanishi H: Lapatinib sensitivities of two novel trastuzumab-resistant HER2 gene-amplified gastric cancer cell lines. Gastric Cancer 17: 450-462, 2014

21. Tang L, Wang Y, Strom A, Gustafsson JA and Guan X: Lapatinib induces p27(Kip1)-dependent $\mathrm{G}_{1}$ arrest through both transcriptional and post-translational mechanisms. Cell Cycle 12: 2665-2674, 2013.

22. Elmore S: Apoptosis: A review of programmed cell death. Toxicol Pathol 35: 495-516, 2007.

23. Motomura M, Kwon KM, Suh SJ, Lee YC, Kim YK, Lee IS Kim MS, Kwon DY, Suzuki I and Kim CH: Propolis induces cell cycle arrest and apoptosis in human leukemic U937 cells through Bcl-2/Bax regulation. Environ Toxicol Pharmacol 26: 61-67, 2008.

24. Ola MS, Nawaz M and Ahsan H: Role of Bcl-2 family proteins and caspases in the regulation of apoptosis. Mol Cell Biochem 351: 41-58, 2011

25. Ouyang L, Shi Z, Zhao S, Wang FT, Zhou TT, Liu B and Bao JK: Programmed cell death pathways in cancer: A review of apoptosis, autophagy and programmed necrosis. Cell Prolif 45: 487-498, 2012.
26. McIlwain DR, Berger T and Mak TW: Caspase functions in cell death and disease. Cold Spring Harb Perspect Biol 7: pii: a026716, 2015

27. Su CC, Chen JY, Din ZH, Su JH, Yang ZY, Chen YJ, Wang RY and $\mathrm{Wu} Y \mathrm{YJ}$ : 13-acetoxysarcocrassolide induces apoptosis on human gastric carcinoma cells through mitochondria-related apoptotic pathways: p38/JNK activation and PI3K/AKT suppression. Mar Drugs 12: 5295-5315, 2014.

28. Osaki LH and Gama P: MAPKs and signal transduction in the control of gastrointestinal epithelial cell proliferation and differentiation. Int J Mol Sci 14: 10143-10161, 2013.

29. Gschwantler-Kaulich D, Grunt TW, Muhr D, Wagner R, Kolbl H and Singer CF: HER specific TKIs exert their antineoplastic effects on breast cancer cell lines through the involvement of STAT5 and JNK. PLoS one 11: e0146311, 2016.

30. Castaneda CA, Cortes-Funes H, Gomez HL and Ciruelos EM The phosphatidyl inositol 3-kinase/AKT signaling pathway in breast cancer. Cancer Metastasis Rev 29: 751-759, 2010.

31. Yu T, Cho BJ, Choi EJ, Park JM, Kim DH and Kim IA: Radiosensitizing effect of lapatinib in human epidermal growth factor receptor 2-positive breast cancer cells. Oncotarget 7: 79089-79100, 2016. 